\title{
Carnets
}

Revue électronique d'études françaises de l'APEF

Deuxième série - 19 | 2020

Petite fabrique d'interprètes

\section{EUPHONIE, d'Hector Berlioz}

De l'Uphonie à l'Utopie

\section{Maria Luísa Malato Borralho}

\section{OpenEdition}

Journals

Édition électronique

URL : http://journals.openedition.org/carnets/11229

DOI : $10.4000 /$ carnets. 11229

ISSN : 1646-7698

Éditeur

APEF

Référence électronique

Maria Luísa Malato Borralho, «EUPHONIE, d'Hector Berlioz », Carnets [En ligne], Deuxième série - 19 |

2020, mis en ligne le 31 mai 2020, consulté le 22 décembre 2020. URL : http://

journals.openedition.org/carnets/11229; DOI : https://doi.org/10.4000/carnets.11229

Ce document a été généré automatiquement le 22 décembre 2020.

\section{(c) (1) (8)}

Carnets est mis à disposition selon les termes de la licence Creative Commons - Atribution - Pas d'utilisation commerciale 4.0 International. 


\title{
EUPHONIE, d'Hector Berlioz
}

\author{
De l'Uphonie à l'Utopie
}

Maria Luísa Malato Borralho

\section{NOTE DE L'AUTEUR}

Cet article s'inscrit dans le cadre de l'axe de recherche "Literatura e Fronteiras de Conhecimento/ Políticas de Inclusão", de l'Instituto de Literatura Comparada Margarida Losa de la Faculté de Lettres de l'Université de Porto, subventionnée par la FCT, dans le cadre du "Programa Operacional Ciência e Inovação (POCI 2010), Quadro Comunitário de Apoio III (UID/ELT/00500/2013 | POCI-01-0145-FEDER-007339).

Dédié à Hermínia Amado Laurel, à Isabel Pereira Leite, à la beauté de la vie et à son impossibilité possible

\section{D'où vient la musique?}

1 Au début, c'était le Son. Pas certainement le Mot. Pas encore la Parole. Mais déjà la partie audible des signes. Quelque chose qui bouge, même si la chose est invisible. Le Son est avant le Verbe, il est avant la création du discours. Si le Cosmos naît du verbe de Dieu et le Mot de la Parole de la mère, l'enfant écoute d'abord la totalité du Son, en effet, premier sens du monde. Le langage naît de cette confiance faite au son, entendu

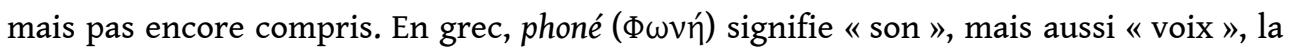
voix des êtres humains, des animaux et des instruments de musique, mais aussi « expression", « langage », et « langue » (Pereira, 1969: 622). Au début, nous sommes tous des étrangers, des barbares qui envahissent le monde. Et nous faisons confiance à la douceur des sons écoutés. Et nous nous méfions des sons qui nous semblent menaçants. Et la saveur douce ou aigre nous dit ce que nous avons besoin de savoir. Le son qui se répète devient après un rythme, une cadence. Les cycles individuels $\mathrm{e}$ 
collectifs, faits de dissolution et renouvellement, ont encore les vestiges du Son et du Silence.

2 Au début, c'était la Musique. Pas certainement l'art de la musique, mais la Musique, encore sans images et sans désir : la musique du cœur de notre mère qui battait près du nôtre, bien avant la vision de cette lumière blanche qui nous aveuglera à la sortie de son ventre. La musique des battements est l'arithmétique des sons. Le "nombre", en grec, se dit "arythmos » (“A $1 \theta \mu$ óc), « énumération » et « durée ». Le verbe, le discours, naitra du nombre. Nous comptons des oranges comme nous racontons des histoires : la succession d'informations présuppose l'absence ou l'exclusion des unités. Aussi en latin, le « numerus » est l'élément reconnaissable dans un ensemble, l'unité, mais aussi, par une métonymie fonctionnelle, la mesure. Et le "numen» est simultanément le mouvement de la tête qui manifeste une volonté individuelle et l'expression dissimulée de la divinité et de la grandeur ineffable (Ferreira, s.d. : 765-766).

\section{L'Euphonia}

3 L'Euphonia, une nouvelle du compositeur français Hector Berlioz, a été publié au long de l'année 1844, éparpillé par plusieurs numéros de la Revue et Gazette Musicale de Paris. Huit ans plus tard, en 1852, l'auteur l'inclura dans un texte plus vaste, Les Soirées de L'Orchestre, dédié «A mes bons amis, les artistes de l'Orchestre de $\mathrm{X}^{* * * *}$, Ville Civilisée ». Dans ce nouveau co-texte (et contexte) de lecture, l'Euphonia devient la dernière soirée des musiciens, la vingt-cinquième. Il est placé pourtant dans un nonlieu, un lieu de lecture, avant l'Épilogue et après la dernière soirée de convive. L'Euphonia est lu quand on sait que le théâtre, le lieu de rencontre des artistes pour leurs soirées, sera fermé "pour cause de réparations", quand s'installe, entre les musiciens, une certaine lassitude s'installe. C'est "notre ami Corsino », un des artistes italiens, qui fermera les soirées littéraires "en lisant une Nouvelle qu'il vient de terminer. On demande " - Silence ! » pour écouter la lecture, mais on doit y écouter un « chœur prodigieux et ce duo qui ne l'est pas moins !...» (1852: xxIv). La 25ème soirée découle alors de ce double non-lieu, le non-lieu de la lecture et le non-lieu de la musique qui doit s'écouter avant la lecture :

On joue, etc., etc., etc., etc.

A peine les premiers accords de l'ouverture sont-ils frappés, que Corsino déroule son manuscrit, et lit ce qui suit avec accompagnement de trombones et de grossecaisse. Nous l'entendons néanmoins, grâce à l'énergie et au timbre singulier de sa voix.

- Il s'agit, messieurs, dit-il, d'une nouvelle de l'avenir. La scène se passera en 2344, si vous le voulez bien. (Berlioz, 1852 : en ligne)

4 J'ai trouvé la traduction portugaise de cette nouvelle en 2012, dans une petite librairie de la ville de Porto, la Livraria Utopia. L'édition était déjà de 2004, de la maison éditrice \& Etc, de Lisbonne. J'ai encore su, bien plus tard, en 2020, qu'une amie à moi, Isabel Pereira Leite, avait passé par là, à peu près à la même époque, et elle avait acheté aussi un exemplaire du même livre, attirée peut-être par la même fragilité de la plupart des choses qui nous semblent belles: sa taille petite, la couverture délicatement doublée par les oreilles du livres, les roses qui rappelaient William Morris, et peut-être une conférence que Morris a prononcé à Town Hall, Birmingham, en 1880, publiée aussi en portugais par la même maison d'édition (Morris, 2007): "Most people live as if the beauty of life were irrelevant or an unaffordable luxury, whereas art and beauty in the 
widest sense, are vital elements in the life Nature intended us to lead " (Morris 1880, en ligne). Morris annonce le sujet bizarre de la conférence : la beauté de la vie, la beauté du monde, condition sine qua non de la vie individuelle et collective. Et pourtant il demande à ceux qui s'étonnent du sujet, quel est le pourcentage de la population des pays civilisés qui s'occupent de cette question radicale, à la racine du plaisir de vivre? Pourquoi ne voit-on pas l'évidence, et l'urgence, de la beauté ?

My message is, in short, to call on you to face the latest danger which civilisation is threatened with, a danger of her own breeding: that men in struggling towards the complete attainment of all the luxuries of life for the strongest portion of their race should deprive their whole race of all the beauty of life: a danger that the strongest and wisest of mankind, in striving to attain to a complete mastery over nature, should destroy her simplest and widest-spread gifts, and thereby enslave simple people to them, and themselves to themselves, and so at last drag the world into a second barbarism more ignoble, and a thousand fold more hopeless, than the first. (Ibidem)

\section{La synchronie}

5 Le narrateur de l'Euphonia ne s'appelle pas Berlioz, ni Corsino, mais Xilef, qui est le palindrome de Felix, le nom latin pour "Heureux ». Même si Berlioz et Corsino nous transportent dans un avenir eutopique, en 2344, les premiers mots de Xilef sont le souvenir d'un plaisir ab initium. Au début de l'Euphonie, la première lettre du narrateur principal Xilef (compositeur, préfet des voix et des instruments à cordes de la ville d'Euphonia), dirigée à son ami Shetland (compositeur, préfet des instruments à vent), est, comme dans la conférence de Morris, la (re)constitution d'un nouvel «omphalos » du monde, après la destruction critique de la contemporanéité. Xilef se bagne dans l'Etna, nombril du monde qui ressemble à la caisse de résonance d'un alto, l'instrument que Berlioz a appris à jouer dans la petite enfance, lui qui ne jouera pas le piano comme tous les autres compositeurs célèbres:

Je viens de me baigner dans l'Etna! ô mon cher Shetland, quelle heure délicieuse j'ai passée à sillonner à la nage ce beau lac frais, calme et pur! Son bassin est immense, mais sa forme circulaire et l'escarpement de ses bords en rendent la surface sonore au point que ma voix parvenait sans peine du centre aux parties $d u$ rivage les plus éloignées. Je m'en suis aperçu en entendant applaudir des dames siciliennes qui se promenaient en ballon à plus d'une demi-lieue de l'endroit où je m'ébattais comme un dauphin en gaieté. Je venais de chanter en nageant une mélodie que j'ai composée ce matin même sur un poème en vieux français de Lamartine, que l'aspect des lieux où je suis m'a remis en mémoire. Ces vers me ravissent. Tu en jugeras : Enner m'a promis de traduire le Lac en allemand. (Berlioz, 1852, en ligne)

6 Lu dans la deuxième édition, à la fin des Soirées de l'Orchestre, l'Euphonie, la nouvelle sur l'avenir, répond à la Première Soirée du livre de 1852, significativement nommée «Le premier Opéra. Nouvelle du Passé (1555)». Le parallélisme entre la Première et la Dernière Soirée est indéniable. La lettre de Xilef à Shetland, écrite en Sicile, le 7 juin 2344, est la transposition, dans l'avenir, de la lettre de Alfredo della Viola à Benvenuto Cellini, écrite à Florence le 27 juillet en 1555, dans le passé :

Il y a deux ans, je formai le plan d'un ouvrage de théâtre sans pareil jusqu'à ce jour, où le chant, accompagné de divers instruments, devait remplacer le langage parlé, et faire naître, de son union avec le drame, des impressions telles que la plus haute poésie n'en produisit jamais. [...] les paroles expriment l'amour, la colère, la 
jalousie, la vaillance ; et le chant, toujours le même, ressemble à la triste psalmodie des moines mendiants. Est-ce là tout ce que peuvent faire la mélodie, l'harmonie, le rythme? N'y a-t-il pas de ces diverses parties de l'art mille applications qui nous sont inconnues? Un examen attentif de ce qui est ne fait-il pas pressentir avec certitude ce qui sera et ce qui devrait être ? (Berlioz, 1852 : en ligne)

La Vingt-Cinquième Soirée reprend le désir de la Cour du noble Bardi, à la renaissance italienne: refaire le mélodrame, le théâtre en musique repris des grecs, le rêve de l'œuvre totale, l'Opéra, qui puisse joindre finalement ce qui a été écarté, la Musique et la Littérature: ne s'agit-t-il pas de «pressentir avec certitude ce qui sera et ce qui devrait être? » (Berlioz, 1852 : en ligne). « La scène se passera en 2344, si vous le voulez bien. ", annonçait Corsino, faisant un clin d'œil au lecteur. L'utopie devient en effet, encore une fois, malgré la projection du futur, qui l'approche de la science-fiction, un croisement d'époques différentes : le passé et l'avenir dans le présent. Tous les temps sont ici à l'Etna. Tous les temps se traduisent, cela veut dire, se trainent. Enner (qui s'écrivait Enneh dans la première version de l'Euphonie, en 1844) est encore l'anagramme du poète Heine, ici traducteur de Lamartine. Et le paysage évoque simultanément les quatre éléments essentiels : le feu, l'eau, la terre et l'air (incarnés par le volcan, le lac, les berges et le ballon), à partir desquels toutes les choses sont faites. L'Etna est simultanément l'image de la première et de la dernière (re)connaissance. L'Euphonia décrite par Berlioz est un Éden complexe, il possède aussi une sortie, un arbre de la connaissance, qui révèle inévitablement aussi l'ignorance de ceux qui se croient heureux. Dès les premières lignes, la tranquillité contraste avec la rage, la proportion avec l'excès, tout comme la Beauté n'exclut pas le sentiment du Sublime :

La nature est si belle autour de moi! Cette plaine où fut Messine est un jardin enchanté ; partout des fleurs, des bois d'orangers, des palmiers inclinant leur tête gracieuse. C'est l'odorante couronne de cette coupe divine, au fond de laquelle rêve aujourd'hui le lac vainqueur des feux de l'Etna. Étrange et terrible dut être cette lutte! Quel spectacle! La terre frémissant dans d'horribles convulsions, le grand mont s'affaissant sur lui-même, les neiges, les flammes, les laves bouillantes, les explosions, les cris, les râlements du volcan à l'agonie, les sifflements ironiques de l'onde qui accourt par mille issues souterraines, poursuit son ennemi, l'étreint, le serre, l'étouffe, le tue, et se calme soudain, prête à sourire à la moindre brise !... (Berlioz, 1852 : en ligne)

\section{Le Beau et le Sublime}

8 Le cratère, "la couronne d'huile de cette coupe divine ", est symboliquement (ici et dans la tradition hermétique) un fond baptismal, un lac, l'œil des dieux, et un lieu de révélation, de communication entre l'extérieur et l'intérieur, l'apparence et l'essence, l'exposé et le caché (cf. Chevalier, 1994 : 397). Il nous semble même que la description de l'Etna reprend et nie simultanément, dans le texte de Berlioz, les termes évoqués par Virgile, dans la Enéide, le contraste brusque entre le port, calme et immense, à l'abri des vents, et l'Etna, qui rugit et éclate et soulève des boules de feu et crache ses propres entrailles (III, 570-577). Ou par Longin, dans le texte traduit par Boileau :

Ainsi nous n'admirons pas naturellement de petits ruisseaux, bien que l'eau en soit claire et transparente, et utile même pour notre usage: mais nous sommes véritablement surpris quand nous regardons le Danube, le Nil, le Rhin, et l'Océan surtout. Nous ne sommes pas fort étonnés de voir une petite flamme que nous avons allumée, conserver longtemps sa lumière pure ; mais nous sommes frappés 
d'admiration, quand nous contemplons ces feux qui s'allument quelquefois dans le ciel, bien que pour l'ordinaire ils s'évanouissent en naissant : et nous ne trouvons rien de plus étonnant dans la nature que ces fournaises du mont Etna, qui quelquefois jette du profond de ses abîmes, Des pierres, des rochers, et des fleuves de flammes. (Longin, 1995, ed. 1701, en ligne)

La présentation de cette lutte primordiale, de ces aspects du Beau et du Sublime de Longin, commenté par Boileau, repris au XVIII siècle par Burke (et après par Kant et Schiller) et relus par Romantisme français, anglais et allemand que Berlioz connaissait bien, ne sont pas certainement le fruit du hasard, puisque les références de l'époque romantique se mêlent aux références classiques, louant l'ordre et le désordre, et l'eutopie et la dystopie émergeantes de la réalité, qu'elle soit chaotique ou paisible.

Because that union of affecting words, which is the most powerful of all poetical instruments, would frequently lose its force, along with its propriety and consistency, if the sensible images were always excited. There is not perhaps in the whole Eneid a more grand and laboured pas- sage than the description of Vulcan's cavern in Etna, and the works that are there carried on. (Burke, $1990: 155$ )

10 L'Etna est un exemple classique et romantique, un lieu commun pour illustrer le concept de Sublime, et il doit être lu comme un espace significatif. Berlioz trace, tout au long du texte, une dichotomie de plus en plus claire entre ceux qui voient, ceux qui écoutent, les initiés qui ont un certain type de connaissances, et ceux qui sont sourds et aveugles à la beauté du monde. Cette dichotomie se retrouve dans les premières lignes, entre ceux qui sont sensibles au sublime du paysage et ceux qui ne le sont pas. Et peutêtre pour cette même raison, ce qui surprend tout de suite le lecteur est la révélation de la solitude du nageur.

Eh bien, croirais-tu que ces lieux jadis si terribles, aujourd'hui si ravissants, sont presque déserts! Les Italiens les connaissent à peine! On n'en parle nulle part; les préoccupations mercantiles sont si fortes parmi les habitants de ce beau pays, qu'ils ne s'intéressent aux plus magnifiques spectacles de la nature qu'en raison des rapports qu'ils peuvent apercevoir entre eux et les questions industrielles dont ils sont agités jour et nuit. Voilà pourquoi l'Etna n'est pour les Italiens qu'un grand trou rempli d'eau dormante, et qui ne peut servir à rien. (Berlioz, 1852 : en ligne)

11 «Eh bien, croirais-tu? » La complicité avec le narrataire naît de la distance entre le narrateur et son entourage, les Italiens avides de commerce et d'industrie, de profit matériel, à Florence ou en Sicile. Il cherche alors, dans le passé ou dans l'avenir, un âge d'or, une Grèce mythique : «il n'y a toujours pas tant de riches en poètes, peintres et musiciens, qu'après la Grèce c'était le deuxième grand temple de l'art, où le peuple luimême en avait le sentiment, où les artistes éminents étaient honorés presque autant qu'ils le sont aujourd'hui dans le nord de l'Europe » (Berlioz, 1852 : en ligne). Celui qui connaît le sublime est donc toujours, dans l'espace ou dans le temps, un homme insolite, supérieur et différent des autres hommes, même quand, en apparence, il jouit de la connaissance en tant que paysan, enfant ou dauphin.

12 Schiller est peut-être, plus que Kant ou même Burke, le philosophe de cette solitude du Sublime, le dramaturge qui souligne cette illisibilité d'un certain type de beauté, presque sauvage et sans doute vitale : le sentiment éprouvé par le voyageur qui touche la fatigue extrême est aussi la vitalité qui l'anime et lui donne l'enthousiasme suffisant pour continuer, leur donnant l'accès au plaisir qu' il est le seul à jouir, face à l'anarchie douteuse du monde moral (Schiller, 1997 : 226). Ce sentiment n'est pas encore une idée : il est seulement un son, une musique. Significativement, Proust appelait cet éblouissement intellectuel un «moment musical». Jean Grenier retiendra cette même 
liaison entre vie et musique quand un jour, à bout de souffle, au sommet d'une montagne, il sentit le paysage s'élargir et des passages des symphonies de Beethoven lui venaient à l'esprit, pour nous imposer la même mélodie et «d'auditeurs nous transformer en acteurs» (Grenier, 1941: 15). L'éblouissement, origine de la philosophie, de la poésie, de la science, voilà ce qu'il nous faut chercher, voilà sur ce qu'on doit parler, parce c'est lui qui nous soutient. Gaston Bachelard, à la fin de $L a$ Dialectique de la Durée, nous parle aussi de cela, de l'importance des investigations sur le rythme, la Rythmanalyse, conçue par un professeur de l'Université de Porto, au Brésil, dont il avait pris connaissance. Pendant des années on a cru à une fiction, puisqu'il n'y a pas une université de Porto au Brésil. Et pourtant la référence cachait le nom de Lúcio Alberto Pinheiro dos Santos, professeur de l'Université de Porto, mis en exil (Baptista, 2010). La nouvelle branche de la science, la Rythmanalyse, serait précisément une "phénoménologie rythmique ", une méthode d'éblouissement systématique qui retrouve les yeux ravis devant les scènes jadis quotidiennes et communes (cf. Bachelard, 1963: VIII, 129). Le philosophe, le scientifique et l'artiste seraient tous d'éternels enfants, curieux et émerveillés par les routines du monde, et, contrairement à l'homme "sclérotique", ils flueraient à travers les rythmes, les métaphores, les allitérations, les valeurs inconnues et les énigmes.

\section{La musique est une rhétorique}

13 Avant le sens, ce que l'animal, l'enfant et l'étranger perçoivent, ce sont les sons et les rythmes. Le rythme est lui-même une rhétorique : il organise l'inventio (un inventaire connotatif, une échelle de sons agréables ou désagréables, pacifiques ou redoutables), il impose une dispositio (un mouvement ambulatoire, respiratoire ou cardiaque qui organise la mélodie et les pauses), il fait partie de l'elocutio (qui relie les formes et les contenus, les intonations et les sentiments). Le rythme est l'expression linguistique structurelle (Benveniste, 1966 : 327). Amorim de Carvalho, tout au long de sa théorie générale de la versification, reliera les rythmes à ce qu'il appelle une tendance physiologique et esthétique (Carvalho, 1987 : II, 11 et passim). Et Henri Meschonnic fonde une anthropologie historique du langage sur le rythme (Meschonnic, 1987: 44). Les preuves d'autorités sont innombrables, mêlant toujours cet éblouissement de la musique au réveil du langage. Étymologiquement, le mot "chiffre" fait référence à "sephiro ", qui, en hébreu, identifie l'acte de dire, comme la première émanation du mystère de Dieu, et cela ne manquera pas d'être souligné dans de nombreux ouvrages anciens sur la philosophie pythagoricienne, le l'alchimie, ou kabbalistique (cf. TryonMontalembert et Hruby, 1999 : 76).

Dans la plupart des langues latines, la «comptabilité » et l'acte de « raconter » ont un lexème en commun : « compter ». D'une certaine manière, ces deux sens sont déjà unis également dans les Mille et une nuits, un récit sur l'enchantement de dire. Pour Xhéhérazade, raconter est à la fois dire et contrôler le rythme de ce qu'elle dit, jour après jour, unité par unité, enchaîner des histoires qui ne veulent pas finir, être finies. D'ailleurs, l'expression « mille et un » n'est pas innocente, bien qu'aujourd'hui, pour un profane, ce n'est qu'une façon de dire «Beaucoup». « 1001 » est un palindrome et il présente un modèle de symétrie qui se répète, avec des variantes toujours plus complexes, dans le schéma de multiplication des nombres premiers (uniquement divisible par lui-même et par l'unité). L'expression séduit les mathématiciens : « 1001 » 
est le résultat de la multiplication de 3 nombres-premiers : 7, 11 et 13. Des nombres qui, à cause des calculs qui ont été faits avec eux, ont fonctionné à partir de 7, comme des nombres magiques, avec des forces intellectuelles et imprévisibles, fruits de la volonté et du hasard, que seuls les experts pouvaient manipuler. Bucky Fuller identifie le nombre 1001 avec ce qu'il appelle le SSRCD, a « Scheherazade Sublimely Rememberable Comprehensive Dividend ", qui condense le savoir scientifique et le savoir magique, les sciences et les arts :

I think the Arabian priest-mathematicians and their Indian Ocean navigators ancestors knew that the binomial effect of 1001 upon the first prime numbers $(1,2$, 3 and 5) did indeed provide comprehensive dividend accommodation of all the permutative possibilities of all the «story-telling-taling-talling» or computational systems of the octave system of integers. (Fuller, ed. 1975 : 772)

Pseudo-Aristote avait déjà tenté de répondre à cette question lorsqu'il suggère la liaison probable entre la musique et le «mouvement », les actions... Serait-il parce que, par nature et depuis l'enfance la plus reculée, nous sommes des êtres qui apprécient le mouvement, se demande-t-il ? Ou parce que, de façon plus diffuse, la consonance est la fusion d'éléments opposés dont nous dépendons également, la répétition et le nouveau? (apud Gevaert / Volgraff, 1988 : 71-73). Berlioz n'est pas un philosophe. Mais en tout cas, il est difficile de séparer dans l'œuvre d'Hector Berlioz, notamment dans l'Euphonie, les réalités qui sont généralement conçues comme antithétiques : l'art de la musique et l'art littéraire, la pensée et le son...

[...] je lui parle... dans son dialecte scandinave : «Mina! sare disiul dolle menos ? doer si men? doer? vare, Mina, vare, vare!» Puis, sa tête inclinée sur mon épaule, nous murmurons doucement nos intimes confidences, et nous parlons des premiers jours. (Berlioz, 1852 : en ligne) ${ }^{1}$

\section{Un besoin d'absolu}

Les biographes de Berlioz se disent perplexes face à la folie de ses projets. La musique qu'il avait dans la tête avant tout, mais elle semblait impossible. Henry Barraud commence la biographie de Berlioz avec une citation du compositeur : " Ma vie est un roman qui m'intéresse beaucoup", ainsi disait Berlioz (Barraud, 1999: 11). Hector Berlioz semble toujours entendre un accord harmonieux entre ses deux démons : l'un qui prêche la rigueur et l'autre l'euphorie. En bref, il cherche la rigueur de l'euphorie, la possibilité des impossibilités. Il vit toujours très proche de l'utopie. Saint-Saëns déclarait que les misères de Berlioz provenaient de la recherche de l'impossible, et de la piété qui en résultait (Elliot, 1959:24) et son biographe Henri Barraud résumait la pensée de Berlioz par un incontrôlable « besoin d'absolu » (Barraud, 1989 : 457).

Pourtant, le père d'Hector Berlioz, Louis Berlioz, lui avait donné une éducation pragmatique. Il lui avait laissé un carnet prouvant par la généalogie de sa famille l'ascendance de quatre siècles de sagesse : le carnet avait le titre Livre de Raison. Mais, pour Hector Berlioz, la généalogie est centrée dans les aventures d'un oncle extravagant, Félix Marmion, voyageur en Europe au service de Napoléon. Et son « livre de raison " semble être plutôt l'Enéide de Virgile, livre où il trouvait clarté et passion, militarisme et aventure (et il sera fidèle à cette lecture de jeunesse dans plusieurs de ses opéras, comme La Prise de Troie et Troyens à Carthage). Sa réalité est littéraire. Même les femmes dont il tombera amoureux se confondent avec les héroïnes littéraires des romans ou des drames qu'il admire. Hector tombera amoureux pour la première fois en 
1815, à 12 ans, par Estelle Duboeuf, jeune voisine, aux chaussures roses. Son nom, Estelle, et ses yeux verts lui rappellent la fée d'une pastorale de Florian, Estelle et Némorin, alors très à la mode (Barraud, $1988: 14$ ). Cette première mémoire de l'amour l'accompagnera aussi toujours : Estelle sera le thème d'une chanson qui deviendra plus tard le motif de la Symphonie Fantastique (1830). Le compositeur terminera ses Mémoires (1865) avec une évocation à "Stelle Montis ", son constant amour. Il la rencontrera à la fin de sa vie, quand elle était déjà une bonne grand-mère à 70 ans, mais elle ne se rappelait plus son petit voisin et niait l'existence de ses chaussures roses (Elliot, 1959 : 25).

Hector avait 23 ans lorsque, le 6 septembre 1827, une troupe de théâtre anglaise fait ses débuts à l'Odéon de Paris pour représenter Hamlet dans la version originale anglaise. Hugo, Balzac, Vigny, Gauthier, Nerval, Sainte-Beuve, Dumas, Michelet, Delacroix, toute la jeune garde était là, pour assister à l'événement, mais Berlioz sera le plus enthousiaste: il quittera le théâtre amoureux d'Ophelia, qui est en effet une actrice irlandaise nommée Harriet Smithson. Il se déclare, Harriet ne le comprend pas. Elle sort de Paris avec un bref commentaire : «Il n'y a rien de plus impossible!» et Berlioz, éperdu d'amour, demandera à son ami Humbert Ferrand : "Comment je parviendrais à être aimé d'Ophélie ?". Comment peut-être impossible un sentiment si réel ? "Oh! Malheureuse! Si elle pouvait concevoir toute la poésie, tout l'infini d'un pareil amour, elle volerait dans mes bras [...]» (Barraud, 1988 : 30). Il la poursuit, habillé en femme, " robe, chapeau, voile vert ", avec les pistolets chargés et deux flacons de laudanum et de strychnine. Il tente le suicide, mais, sur une route de Damas, il retrouve la raison. Il composera Songe d'une nuit de Sabatt où il fermera Harriet Smithson avec les vêtements d'une sorcière, chevauchant un balai. Mais on la retrouve encore à la base de la Symphonie Fantastique, " un nouveau genre musical». Berlioz décrira les personnages à Ferrand: "le jeune artiste en proie aux espoirs et aux déceptions d'un amour malheureux ; la femme aimée, fixée dans un contour mélodique obsédant ; l'illusion du bonheur dans la paix des champs ; le tourbillon d'un bal ; le meurtre de la femme aimée et la marche au supplice de l'amant meurtrier ; l'orgie d'une nuit de sabbat où se vautre l'objet de son amour rendu à sa condition de courtisane" (Barraud, 1988 : 35).

\section{Eutopie et Dystopie}

19 On n'est pas loin des personnages de l'Euphonia: déception de Xilef, le jeune artiste ; obsession pour Mina, la femme bien-aimée, l'art; livraison de Xilef au travail; retrouvailles de Xilef et Mina lors d'un bal à Euphonia; mort physique et marginalisation sociale de la femme bien-aimée, suicide de l'artiste Xilef... Euphonia semble encore reproduire l'argument du Songe d'une nuit de Sabatt ou de la Symphonie fantastique. Le motif est vraiment une idée-fixe, obsessionnel. Qui peut être à la base des personnages de ses «symphonies à clé »? Parle-t-il de Estella-Estella ou d'OphélieHarriet? Et qui est Xilef? Berlioz ? Ou son oncle Félix Marmion, le voyageur qui flirtait vaguement avec la jeune Estelle Duboeuf (Barraud, 1989 : 14)?

En 1829, il rencontre la pianiste Camille Moke, au domicile de son ami Ferdinand Hiller. Berlioz préparait à l'époque un prélude à $L a$ Tempête pour honorer sa passion pour Shakespeare : Camille fait le rôle d'Ariel. La mère de Camille garde bien sa fille pour un bon mariage, mais en juin 1830, Berlioz s'enfuit avec Ariel. Ils sont pris à Vincennes, mais la valeur de la fiancée semble perdue. La mère se résigne. En plus, le 21 août 1830, 
Berlioz reçoit le premier prix du Concours de Rome. Il est contraint de se rendre en Italie et les amants se disent à bientôt en plein désespoir. Quelques jours après, Ariel épousera l'industriel Pleyel, un très célèbre constructeur de pianos. Dans l'argument d'Euphonie, dix ans plus tard, Mina est encore Ariel-Camille et Ophélie-Harriet. Il y a dans le récit une indéniable catharsis. A la fin, quand Mina et ses invités sont écrasés par les murs du moulin (un énorme piano-orchestre), Xilef s'exclame comme un fou :

Eh bien! Mina, qu'as-tu donc, chère belle, à t'emporter de la sorte? Ton corset d'acier te serrerait-il trop? Prie un de ces messieurs de le délacer, ils en ont l'habitude ! Et ton hippopotame de mère, comment se trouve-t-elle? Je n'entends plus sa douce voix!» En effet, aux cris d'horreur et d'angoisse, sous l'étreinte toujours plus vive des cloisons d'acier, vient de succéder un bruit hideux de chairs froissées, un craquement d'os qui se brisent, de crânes qui éclatent; les yeux jaillissent hors des orbites, des jets d'un sang écumant se font jour au-dessous du toit du pavillon; jusqu'à ce que l'atroce machine s'arrête épuisée sur cette boue sanglante qui ne résiste plus. (Berlioz, 1852 : en ligne)

21 L'histoire d'Euphonia se confond avec la Symphonie Fantastique de Berlioz où la douceur alterne avec le déchirement. C'est pourquoi il est également difficile de distinguer le mouvement qui relie le réel à l'idéal, l'eutopie à la dystopie, l'euphonie à la cacophonie. Bien qu'à des extrémités opposées, le monde d'Hector Berlioz, dans l'agenda de l'orchestre ou dans l'œuvre littéraire, circule entre des vases communicants. Ce que Berlioz recherche, c'est peut-être un lieu de rencontre. Et c'est peut-être pour cela qu'il serait intéressant de poser ici la question de l'utopie (ou " uphonie ») dans le texte de Berlioz. Les Euphoniens aussi, comme les utopistes d'une certaine manière, sont définis en grande partie par la négation plutôt que par la perfection, s'écartant même de quelques ambiguïtés de l'œuvre de Thomas Morus. Voyons-le :

a. Malgré son relief, l'Euphonie, significativement située en Allemagne, aux montagnes de Hartz, n'est pas inaccessible. Les Euphoniens regrettent même ne pas être visités et connus par d'autres peuples (Berlioz, $2004: 38$ ).

b. L'Euphonia n'est pas une structure autonome, mais une partie d'une structure politique plus étendue qui soutient les artistes : «L'empereur d'Allemagne fait tout, d'ailleurs, pour rendre aussi heureux que possible le sort des Euphoniens. Il ne leur demande en retour que de lui envoyer deux ou trois fois par an quelques milliers de musiciens pour les fêtes qu'il donne sur divers points de l'empire » (Berlioz, 1852 : en ligne).

c. L'Euphonia n'est pas un pays parfait : « On peut la considérer comme un vaste conservatoire de musique, puisque la pratique de cet art est l'objet unique des travaux de ses habitants » (Berlioz, 1852: en ligne). Pour cette raison, les Euphoniens voyagent beaucoup pour augmenter les connaissances qu'ils croient ne pas posséder.

d. L'Euphonia n'est pas une démocratie ou un régime qui promet le bonheur. Ses lois sont la discipline du travail, la récompense du mérite, le désir de s'améliorer et le mépris de l'ornement : « Il est inutile de dire qu'Euphonia est gouvernée militairement et soumise à un régime despotique » (Berlioz, 1852 : en ligne).

e. La qualité des citoyens est l'équilibre entre la spécialité de chacun et l'utilité de tous. Tous doivent connaître les différents domaines musicaux et avoir une connaissance approfondie des poètes, anciens et modernes. Personne ne peut maintenir une vision égoïste ou hiérarchisée de l'harmonie musicale, d'une voix ou d'un type d'instrument (Berlioz, 1852 : en ligne). Un temps sacré, qui est aussi un temps musical, est marqué par un orgue à vapeur, dont le langage ne peut être pleinement compris que par les Euphoniens.

f. La qualité de l'art est le mépris de l'inutile. Les choristes ne peuvent pas bouger leur corps pour accentuer le rythme; les entrées doivent être attaquées en masse ; tous les musiciens et chanteurs sont entraînés pour obtenir le silence, un silence si profond qu'on devrait 
entendre un insecte entre les pauses des notes. Tout vise la force de ce sentiment : les salles de concerts pouvaient avoir une capacité de 10 mille interprètes pour un public de douze mille auditeurs (Berlioz, 1852 : en ligne). L'hymne composé par Shetland aura 6 mille voix, cent familles de clarinettes et saxophones, cent familles de flûtes, quatre cents violoncelles et trois cents harpes.

\section{8. « Il n'y a rien de plus impossible !»}

Berlioz a toujours poursuivi le monde qu'il représentait en Euphonia, même si les producteurs de ses spectacles coupaient le nombre de musiciens ou les échecs financiers se succédaient. Un requiem présenté à Paris a réuni six cents interprètes : " dieux et demi-dieux chantant dans le chœur comme des mortels », ainsi le décrivait Berlioz (apud Barraud, 1989: 136). Et parfois la vie nous donne ce que nous lui demandons. "Il n'y a rien de plus impossible!", avait déclaré Harriett Smithson à Hector Berlioz avant de retourner à Londres. Et pourtant, Harriett épousera Hector, quelques années plus tard. Et Ophélia remplira la vie d'Hector, maintenant de désirs, de jalousie et de suspicion. La mort la libère enfin, le 3 mars 1854, et Liszt console Hector, essayant de lui expliquer cet amour impossible: «Elle t'a aimé, tu l'as aimée, tu l'as chantée, sa tâche était accomplie ». Mais Berlioz est comme Xilef :

Nous souffrons tant, nous autres enfants de l'art aux ailes de flamme; nous, élevés sur son giron brûlant ; nous, dont les passions poétisées labourent impitoyablement le cœur et le cerveau pour y semer l'inspiration, cette âpre semence qui doit les déchirer encore quand ses germes se développeront !... Nous mourons tant de fois avant la dernière !... Shetland ! Shetland ! je l'aime !... je l'aime, comme tu l'aimerais toi, si tu pouvais ressentir un amour autre que celui dont tu m'as fait la confidence! Et pourtant, malgré la grandeur et l'éclat de son talent, Mina m'apparaît souvent comme une organisation vulgaire. Te le dirai-je? Elle préfère le chant orné aux grands élans de l'âme; elle échappe à la rêverie; elle entendit un jour à Paris ta première symphonie d'un bout à l'autre sans verser une larme; elle trouve les adagios de Beethoven trop longs !... (Berlioz, 1856 : en ligne)

Il se remariera, avec une ancienne amante, Marie Recio : il le faut, selon lui. Tout le monde a un Livre de raison. Il aura encore le temps d'écrire ses Mémoires. Et à la fin de sa vie, les honneurs se succèdent : il lira encore les critiques enthousiastes de la presse allemande, belge (et même française), il écoutera les ovations du public (même pour ses opéras), la Légion d'honneur (bien sûr), et la présidence de l'Académie des Beaux-Arts... La vie est un phénomène étrange, se dit-il, parce que, à la fin de sa vie, il ne compose plus. Depuis janvier 1869, Berlioz vit dans un état léthargique. Par testament, il laisse au Conservatoire de Paris ses partitions et manuscrits. À trois bons amis, "mon Shakespeare anglais», "mon Virgile latin», "mon Paul et Virginie annoté de ma main ». Et à Estelle Duboeuf, un revenu annuel de seize cents francs: " comme un souvenir des sentiments que j'ai éprouvés pour elle toute ma vie... » (Berlioz, 1868). Ses derniers mots à l'arrivée de la mort sont ironiques: «...Enfin, on va jouer ma musique ». Il décède à Paris le 8 mars 1869.

Le 11 avril 2020, la veille du dimanche de Pâques, j'ai reçu de mon amie Isabel un essai publié au Brésil, sur le lieu de la musique dans un temps dystopique. Il commençait précisément avec une évocation de l'Euphonia, ce texte rare de Berlioz (Leite, 2020 : en ligne). Elle me le dédiait, ne sachant pas que j'avais acheté le même livre à la même petite librairie, et que, simultanément, j'écrivais cet essai d'hommage à Hermínia 
Amado Laurel. Fermées chez nous, à cause d'une pandémie qui passera comme d'autres, on pense " à nos bons amis ", aux liens impossibles de la beauté toujours possible.

«Il n'y a rien de plus impossible !» ... Au moment où les agents déposent le cercueil dans le corbillard, les chevaux prennent la bride. Ils traversent la ville jusqu'à la porte du cimetière de Montmartre. Et ce sont eux, sans un cocher, qui ainsi conduisent Hector Berlioz à sa dernière adresse.

\section{BIBLIOGRAPHIE}

BACHELARD, Gaston (1963). La Dialectique de la Durée. Paris : Presses Universitaires de France.

BAPTiSTA, Pedro (2010). o Filósofo Fantasma. Sintra : Editora Zéfiro.

BARRAUD, Henry (1989). Hector Berlioz. Paris: Fayard.

BERLIOZ, Hector (1844). « Euphonia ou La Ville Musicale », in Revue et Gazette Musicale de Paris. Paris : Au Bureau d'Abonnement, 97, rue Richelieu. [Disponible le 2/4/2020] <URL : https:// archive.org/details/revueetgazettemu1844pari/page/n5/mode/2up>

BERLIOZ, Hector (1852). Les Soirées de l'Orchestre. XXVe Soirée : « Euphonia ou La Ville Musicale ». [Disponible le 2/4/2020] <URL : http://www.hberlioz.com/Writings/ SOindex.htm>

BERLIOZ, Hector (1868). Testament. Site Hector Berlioz. [Disponible le 22/4/2020] <URL : http:// www.hberlioz.com/Works/testament-f.htm>

BERLIOZ, Hector (2004). Eufonia ou A Cidade Musical. Lisboa : Editora Etc.

CARVALHO, Amorim de (1987). Teoria Geral da Versificação, 2 vols. Lisboa : Império.

ElLiot, J. H., Berlioz (1959). Berlioz, London/ New York: J. M. Dent and Sons/ Farrar, Strauss and Cudaby.

FERreIRA, A. Gomes (s.d.). Dicionário de Latim-Português. Porto : Porto Editora.

FULLER, R. Buckminister (1975). Explorations in the Geometry of Thinking. Synergetics, New York/ London : MacMillan/ Collier MacMillan.

GRENIER, Jean (1941). Inspirations Méditerranéennes. Paris : Gallimard.

Leite, Isabel Pereira (2020). « Lugares da música numa distopia : a pandemia de 2020 », Convenit Internacional, São Paulo, n. 34. Set-Dez., 1-12. ISSN 1517-6975. [Disponible le 22/4/2020] < URL : http://www.hottopos.com/convenit34/index.htm>

LONGIN (1995). Traité du sublime ou du merveilleux dans le discours (trad. Boileau). Paris : Le Livre de Poche, Librairie Générale Française, 1995. Reproduction de l'édition de 1701. [Disponible le 2/4/2020] <URL : http://sublime.nancyholt.com/Longinus/ index.html>

MESCHONNIC, Henri (1982). Critique du rythme. Anthropologie Historique du Langage. Lagrasse : Verdier.

MORRIS, William (2007). A beleza da vida. Lisboa : Etc. 
MORRIS, William (1880). The beauty of life. [Disponible en ligne le 2/4/2020]. <URL : https:// edu.glogster.com/glog/pdf-download-the-beauty-of-life-william-morris-and-the-art-of-de/ 362kki8f4us>

PereiRA, Isidro (1969). Dicionário Grego-Português e Português-Grego. Porto : Apostolado da Imprensa. GEVAERT, V.-A. ; VOLGRAFF, J.-C./ eds. (1988). Les problèmes musicaux d'Aristote, Gant : Biblio Verlag. SCHILLER, Friedrich (1997). Textos sobre o Belo, o Sublime e o Trágico. Lisboa : Imprensa Nacional-Casa da Moeda.

TRyon-Montalembert, R. e Hruby, K. (1999). A Cabala e a Tradição Judaica. Lisboa : Edições 70.

\section{NOTES}

1. L'évocation du danois de Mina, langue d'Hamlet et d'Ophélie, n'est qu'une stratégie musicale de Berlioz, la reconstruction d'un rythme et d'une mélodie pour un sentiment. Nous remercions à Gonçalo Villas Boas e à Caroline Meyer leurs efforts de traduction...

\section{RÉSUMÉS}

Hector Berlioz a publié en 1844 un petit récit où il imagine une ville de l'avenir régit par des principes musicaux, L'Euphonia. Republiée en 1852, dans un autre contexte critique, faisant partie des Soirées de L'Orchestre, il occupera la dernière soirée, après l'annonce de la fermeture du théâtre ("pour cause de réparations») et avant l'épilogue de l'œuvre. Les "réparations » gagnent ainsi un symbolisme collectif, puisque l'ordre sociale est aussi une harmonie musicale. L'euphonie et l'eutopie s'imposent, à la vie, à la littérature et à la musique mais elles semblent indissociables de la dystopie, l'utopie étant la représentation de l'absence de lieu, peut-être le seul lieu où l'on peut recréer le monde?

In 1844, Hector Berlioz published a small novel about a future land ruled by musical principles, Euphonia. Remake in 1852, in a different critical context, the novel is the last part of another book, Les Soirées de l'Orchestre. "L'Euphonie" is the last meeting when the theatre was already close « for works » and before the Epilogue of the book. These "works" seems to have a collective intention, since the social order is also a musical harmony. Euphonia and eutopia impose themselves on life, on literature and on music, but they seem voted to dystopia, utopia the representation of a non-place, perhaps the only place capable to recreate the world.

\section{INDEX}

Keywords : utopia, community, literature, music, Berlioz (Hector)

Mots-clés : utopie, communauté, littérature, musique, Berlioz (Hector) 


\section{AUTEUR}

MARIA LUÍSA MALATO BORRALHO

Instituto de Literatura Comparada

Université de Porto

mlmalato[at]gmail.com 